\title{
Agilität, Ambidextrie und organisationale Veränderungskompetenz. Rudi Wimmer über Erbe und Zukunft des Change Managements
}

\author{
Rudi Wimmer ${ }^{1}$ Falko von Ameln² (iD \\ Online publiziert: 22. Mai 2019 \\ ๔ Der/die Autor(en) 2019, korrigierte Publikation 2020
}

\section{Zusammenfassung}

Univ.-Prof. Dr. Rudi Wimmer ist Professor für Führung und Organisation am Institut für Familienunternehmen der Universität Witten/Herdecke. Von 2013 bis 2016 Vizepräsident dieser Universität. Gründer und Partner der osb international AG. Mitglied in Aufsichtsräten unterschiedlicher familiengeführter Unternehmen. Wimmer ist einer der Vordenker der systemischen Organisationsberatung und hat zu diesem Thema zahlreiche Publikationen veröffentlicht.

Schlüsselwörter Ambidextrie · Change Management · Führung · Interne Beratung · Komplexität · Organisationsberatung $\cdot$ Organisationsentwicklung $\cdot$ Organisationstheorie $\cdot$ Selbstorganisation $\cdot$ Systemtheorie

\section{Agility, ambidexterity and organizational change capacity. Rudi Wimmer on the heritage and future of change management}

\section{Abstract \\ 1 Wie bist du zum Thema Organisation und Organisationsveränderung gekommen und was hat dich an diesem Thema fasziniert?}

Univ. Dr. Rudi Wimmer is Professor of Leadership and Organization at the Institute for Family Business of the University of Witten/Herdecke. From 2013 to 2016 Vice President of this University. Founder and partner of osb international AG. Member of supervisory boards of various family-owned companies. Wimmer is one of the pioneers of systemic organizational consulting and has published numerous publications on this topic.

Keywords Ambidexterity $\cdot$ Change management $\cdot$ Complexity $\cdot$ Internal consulting $\cdot$ Leadership $\cdot$ Organization development $\cdot$ Organization theory $\cdot$ Organizational counseling $\cdot$ Self-organization $\cdot$ Systems theory

Ich habe in Wien Verfassungs- und Staatsrecht und Verwaltungsrecht sowie allgemeine Staatslehre studiert und da 1970 promoviert. Von daher war ich mit Organisationsfra-

Univ.-Prof. Dr. Rudi Wimmer

rudolf.wimmer@osb-i.com

PD Dr. Falko von Ameln

info@vonameln.net

1 osb Wien Consulting GmbH, Volksgartenstraße 3/1.DG, 1010 Wien, Österreich

2 Leipziger Str. 9, 26506 Norden, Deutschland gen, speziell in der öffentlichen Verwaltung, intensiv befasst. Während meines Studiums, Ende der 60er-Jahre, hatte ich dann eine sehr befreiende Erfahrung mit der gruppendynamischen Trainingsgruppe. Das war der Einstieg in diese für mich neue Welt der Auseinandersetzung mit sozialen Prozessen. Auf dieser Basis habe ich dann beschlossen, eine intensivere gruppendynamische Ausbildung zu machen. Gleichzeitig war ich eingebunden in diverse demokratiepolitische Reformbewegungen als Teil der 68er-Bewegung in der Schule, der öffentlichen Verwaltung oder in der gewerkschaftlichen Bildungsarbeit, wodurch ich auf einer sehr praktischen Ebene mit Organisationsfragen konfrontiert wurde. Die gruppendynamische Ausbildung und die Auseinandersetzung mit diesen Denkkonzepten, aber auch mit den sozialen Interventionsformen, haben die Hoffnung genährt, einen konkreten Beitrag zum strukturellen Wandel in verschiedenen gesellschaftlichen Bereichen leisten zu können. Von daher war es naheliegend, das Geschehen 
in der Gruppe einzubetten in Organisationszusammenhänge und zu schauen, ob der Einbau von Teamverhältnissen in Organisationen tatsächlich Veränderungen anstoßen kann.

\section{Was machst du heute in der Begleitung von Veränderungsprozessen anders als vor 20 Jahren?}

Die Konsequenz aus den Scheiternserfahrungen mit vielen reformorientierten OE-Projekten der 70er Jahre hat dazu geführt, dass wir Themen des organisationalen Wandels anders angegangen sind. Die Kernfrage, die uns dabei beschäftigt hat, war nicht mehr in erster Linie, wie man in Organisationen emanzipatorische Prozesse in Gang setzen kann, die den Einzelnen aus dem Hierarchiekontext befreien, sondern wir haben sehr viel genauer zu erfassen versucht, warum der jeweilige Status quo unter Veränderungsdruck geraten ist. Dabei haben wir erkannt, dass es ganz unterschiedliche Ansätze braucht und dass der traditionelle Lewin'sche Dreischritt vom Aufeisen, die Dinge neu organisieren und dann wieder stabilisieren, für Veränderungsprozesse sichtlich zu grob und den Komplexitätsverhältnissen des organisationalen Geschehens nicht mehr angemessen ist. Und wir haben sauberer unterschieden zwischen den Veränderungserfordernissen auf der Seite der Organisation und den Veränderungsbedarfen auf der Seite der beteiligten Akteure. Diese Unterscheidung hat das Veränderungsverständnis und das Nutzen unterschiedlichster Beteiligungsmöglichkeiten als Interventionsrepertoire ziemlich angereichert.

\section{Du bist einer der wenigen, die in der Tiefe mit der OE-Tradition und mit systemtheoretischen Beratungsansätzen vertraut sind, und das sowohl aus theoretischer Perspektive als auch aus der Sicht der Beratungspraxis. Worin liegen aus deiner Sicht Erkenntnisse der OE- Tradition, die für Theorie und Praxis heute noch bedeutsam sein können?}

Da möchte ich vor allem drei Punkte herausgreifen. Zum einen sind durch die Tradition der OE sehr viel gezieltere, professionell angeleitete Kommunikationsaktivitäten in das organisationsinterne Geschehen integriert worden. Die traditionellen bürokratisch-hierarchiebetonten Organisationsverhältnisse sind ja prinzipiell auf Möglichkeiten der wechselseitigen Koordination ausgerichtet, die den Kommunikationsaufwand gering halten und somit Kommunikation einsparen. Durch die OE ist eine ganz andere Perspektive auf diese Kommunikationserfordernisse in unsere
Organisationen hineingekommen, nämlich die Erkenntnis, dass intensivere Auseinandersetzungen um unterschiedliche Perspektiven in den Entscheidungsfindungsprozessen eine unverzichtbare Ressource darstellen.

Ein zweiter Punkt ist etwas, das ich den kybernetischen Steuerungsmodus nenne. Heute hat man sich von linearen Beeinflussungsprozessen im Sinne von ,command and control" zumindest teilweise verabschiedet. Man hat gesehen, dass man sinnvollerweise nicht davon ausgehen kann, dass die Organisation 1:1 auf Führungsimpulse im Sinne einer Befolgung reagiert, sondern dass man damit unterschiedlichste Reaktionen auslöst und dass es klug ist, diese Reaktionen zu beobachten, aufzugreifen, sie zu diagnostizieren, um aus der Diagnose heraus nächste Impulse zu setzen. Diese Ersetzung einer linearen Kausalitätsvorstellung durch ein kybernetisches Denken in zirkulären Kausalitäten ist ein ganz wichtiger Impuls der OE.

Der dritte Punkt ist die Bedeutung von Feedbackschleifen und Prozessen der Selbstreflexion. Auch das ist eine wesentliche Erkenntnis der Gruppendynamik- und OE-Tradition. Dass es für die Bearbeitung gewisser Störfaktoren in Organisationen wichtig ist, in eine Metaebene zu gehen, gemeinsam hinzuschauen, was los ist, Feedbackprozesse zu ermöglichen.

\section{Was ist aus deiner Sicht die am meisten unterbewertete Erkenntnis der OE- Tradition?}

Wenn man genauer hinschaut, ist über die Jahre in Organisationen vieles aus der OE-Tradition in Organisationen wirksam geworden. Gleichzeitig habe ich den Eindruck, dass das der OE zugrunde liegende organisationale Entwicklungskonzept immer noch nicht wirklich im Denken der Verantwortungsträger verankert ist. Unsere Organisationen haben in den Jahrzehnten seit dem zweiten Weltkrieg in Auseinandersetzung mit den veränderten Umwelten deutlich höhere Komplexitätsverhältnisse im Inneren aufgebaut. In vielen Fällen sind aber Einstellungen und Denkkonzepte nicht mit diesem Komplexitätszuwachs mitgewachsen. Vielfach gibt es zu wenig Wissen darüber, was es braucht, um die organisationsintern gesteigerte Komplexität sinnvoll zu bearbeiten und nachhaltig auf ein höheres Level der Befassung mit Komplexität zu heben. Dass es auf der Personenseite Personalentwicklungskonzepte für den Kompetenzaufbau braucht, ist in Organisationen akzeptiert. Ein vergleichbares Entwicklungsverständnis mit Blick auf die Kompetenzsteigerung auf einer Organisationsebene, also das, was man in der Literatur mit ,organizational capability“ meint und das man in der OE sozusagen „mit der Muttermilch aufsaugen" kann, ist in unseren Organisationen aus meiner Sicht nach wie vor nur sehr rudimentär 
vorhanden. Dafür braucht es ein weniger instrumentelles Verständnis von Organisation, andere Formen der Entscheidungsfindung, der Kooperation, der Koordination, andere Praktiken, aber auch andere mentale Modelle der Führung.

5 In Deinen Publikationen entwirfst Du ein differenziertes systemtheoretisches Modell von Organisationen als Grundlage für eine organisationstheoretisch informierte Beratung. In der Praxis des Change Managements, so schreibst du in einer deiner Publikationen, ringen wir dagegen „,im Umgang mit der Bewältigung organisierter Komplexität [...] immer noch mit der erstaunlichen Differenz zwischen der beobachtbaren sprachlichen Fassung der eigenen organisationalen Realität und den tatsächlichen bereits in Gang gesetzten Strukturen und Prozessen, die längst einen anderen Typus von Organisation spiegeln. Die Praxis ist unserem alltäglichen Denken über Führung und Organisation in der Regel weit voraus." Inwieweit müssen wir unsere mentalen Modelle von Organisationen, die unserem Change Management implizit zugrunde liegen, weiterentwickeln?

Wir leben ja in einer Phase, die diese Einschätzung besonders provoziert. Man kann das gut daran zeigen, dass viele Unternehmen derzeit durch die Digitalisierung unter einem enormen Innovationsdruck stehen, der Organisationen vielfach unter die bislang nicht gekannte Anforderung stellt, sich geschäftlich radikal neu $\mathrm{zu}$ erfinden und gleichzeitig auch im bestehenden Geschäft ein erhebliches Maß an Veränderungen anzuschieben. Inzwischen gibt es ja an vielen Stellen Lösungsversuche, um diese radikale Innovationsproblematik in irgendeiner Weise bearbeitet zu bekommen. Wir diskutieren unter der Überschrift „Ambidexterity“ Organisations- und Führungslösungen, die diese zum Teil sehr gegensätzlichen Veränderungsherausforderungen Stabilität und Wandel gleichzeitig bearbeiten können.

Die heute sehr favorisierten Agilitätsinitiativen stehen vor ähnlichen Herausforderungen. Für ernsthafte Lösungsaktivitäten in all diese Richtungen braucht es eine andere Organisationsdenke als bisher. Wir müssen über Organisationsdesigns nachdenken, die durch eine gut durchdachte Orchestrierung der Verbindung der Steuerungsmodi „Exploitation“ und „Exploration“ eine gemeinsame Weiterentwicklung in die digitale Welt erwartbar machen können.
6 In einem Interview mit der Zeitschrift "Organisationsentwicklung" hast du darauf hingewiesen, dass unsere mentalen Modelle von Wandel sehr durch westliche Vorstellungen geprägt sind. Du sprichst dich für die chinesische Vorstellung des Wandels als Mitschwimmen im Situationspotenzial der Umweltveränderung aus, nämlich „die Energie des Feldes klug zu nutzen, keine direkten Zielsetzungen auszusprechen und zu verfolgen. Denn das Wasser fließt von oben nach unten, und da geht es darum, genau diese Bewegung, die ohnehin in jede Situationen eingebaut ist, intelligent zu nutzen und in die eigene Richtung zu lenken". Wie könnte das für Change Management konkret aussehen, wo ja oft der Fluss der eigenen Pfadabhängigkeit in eine andere Richtung fließt als der Fluss des evolutionär Sinnvollen?

Die Voraussetzung für einen solchen Wandel der mentalen Modelle ist die Verabschiedung von unseren klassischen Planbarkeitsvorstellungen. Wir haben ja auch im ChangeManagement immer noch die Tendenz, relativ genaue Konzepte für den erwünschten Zielzustand zu entwickeln. Und man ist dann immer fürchterlich enttäuscht, dass die Dinge ganz anders kommen als man gedacht hatte. Für mich ist eine wichtige Begleiterscheinung des fernöstlichen Denkens, dass man sich von solchen Planbarkeitsvorstellungen weitestgehend verabschiedet und sich auf eine, wie du das ja auch richtig nennst, tendenziell evolutionäre Entwicklung verlassen muss.

Wir müssen die Veränderungsenergien, die in alle etwas komplexen Verhältnisse eingebaut sind, zur Entfaltung bringen. Das heißt, dass man fundierte Lernkonzepte baut, um immer wieder neue Aspekte, die im Prozess auftauchen, gezielt zu bearbeiten und für die Weiterentwicklung des nächsten Iterationsschritts produktiv werden zu lassen. Dieses iterative Vorgehen ist für mich eine inzwischen weitgehend akzeptierte, wunderbare Weiterentwicklung unserer klassischen Change-Vorstellungen. Und diese Erfahrungen haben dazu geführt, sich vom sogenannten ,Wasserfallprinzip“ zu verabschieden und das zu entwickeln, was heute unter dem Begriff der Agilität sinnvollerweise diskutiert wird. 
7 Die Systemtheorie ist für die Beratercommunity heute neben der Organisationspsychologie die zentrale Referenzwissenschaft. Wie erklärst Du einem Kunden den Nutzen eines systemtheoretischen Zugangs zum Thema Veränderung?

Vielfach ist das mentale Modell von Auftraggebern, dass externe Berater in ihrem Auftrag in der Organisation die gewünschten Veränderungen anstoßen können, ohne dass die Auftraggeber sich selber zu sehr in diese Prozesse involvieren müssen. Als Berater ist man zu Beginn solcher Prozesse gefordert, zu verdeutlichen, dass organisationaler Wandel letztlich bei einem Wandel der Führungskräfte, ihres Verhaltens und ihrer Formen sich einzubringen, beginnt. Ich sage immer: „Der Wandel der Organisation beginnt mit dem Wandel der Führung." Und diese Selbstbezüglichkeit des Change-Prozesses, dass organisationaler Wandel immer einen Selbstwandel aller Beteiligten impliziert, ist etwas, das man mit einem systemtheoretischen Denkkonzept und -repertoire von Organisation, Führung und Veränderung leichter abbilden kann als mit den üblichen linearen Konzepten der Gestaltung von Organisationen.

\section{Die Rolle von Führung bei der} Sicherung der Zukunftsfähigkeit und Veränderung von Organisationen ist eines deiner zentralen Themen. Vision und Commitment des Top Managements sowie die Bereitschaft, die eigene Rolle kritisch zu reflektieren, erweisen sich sowohl in der Praxis als auch in Studien immer wieder als zentrale Engpässe bei der Veränderung von Organisationen. Wie gehst du in Beratungsprozessen vor, um die Entscheidungsträger bei der Reflexion ihrer Rolle im Veränderungsprozess zu unterstützen?

Das ist eine - auch auf der persönlichen Ebene - sehr, sehr heikle Schlüsselfrage. Für mich ist das immer eine wichtige diagnostische Prüfschleife am Beginn eines Change-Prozesses, ob ich den Eindruck habe, dass sich die Verantwortungsträger auf einen solchen Prozess des Sich-selbst-mitEinbeziehens in das Geschehen einlassen wollen. Das ist ja überhaupt nicht garantiert. Aus so einer impliziten Beauftragung zur Selbstimmunisierung der Topverantwortlichen herauszukommen, ist aber der erfolgskritische Schlüssel. Wenn man das nicht schafft, sollte man von Change-Prozessen besser die Finger lassen. Ich gehe an der Stelle mit den Auftraggebern der Frage nach, was es an Führungsleis- tungen an der Spitze der Organisation braucht, damit das Change-Vorhaben eine Chance hat. Wenn man so eine SollIst-Differenz auf der Ebene „Was braucht es im Führungssystem?" auf dem Tisch hat, dann wird es für die Beteiligten schon sehr schwierig, sich dem total zu entziehen. Meiner Erfahrung nach entsteht dann auch ein Verständnis dafür, dass solche Change-Prozesse große Unsicherheit in der Organisation auslösen. Wenn die Frage ist, wie dieses Maß an Unsicherheit bewältigbar gemacht werden kann, verstehen die Entscheidungsträger dann schon, dass es nicht erfolgversprechend ist, wenn man das einfach hineindrückt in die Organisation oder die Berater diejenigen sein sollten, die diese Unsicherheit absorbieren sollen. Die Bewältigung des mit Change-Prozessen verbundenen zusätzlichen Ausmaßes an Irritationen und Unsicherheit kann eben im Wesentlichen nur von der Unternehmensspitze geleistet werden.

9 Angesichts von Fachkräftemangel und Wertewandel in der Gesellschaft ist immer häufiger zu beobachten, wie Organisationen sich zunehmend um die Bedürfnisse der Mitarbeiter/ innen herumzubauen scheinen Stichworte wären Flexibilisierung von Arbeitszeiten, Entformalisierung der Arbeitskultur, Aufgabenneuverteilung nach Interesse oder Wellnessangebote am Arbeitsplatz. Rückt nun doch der von Luhmann ausgeklammerte Mensch in den Mittelpunkt der Organisation?

Natürlich ändern diese Entwicklungen grundsätzlich nichts daran, dass das Soziale eine Realität sui generis und das Psychische nicht Teil des Organisationsgeschehens ist. Aber durch die Weiterentwicklung der Komplexitätslevels in Organisationen ändert sich der Stellenwert des Beitrages dramatisch, den jeder Einzelne für die erfolgreiche Bearbeitung der Herausforderungen von Organisationen hat. Der tradierte psychologische Vertrag zwischen der Organisation und den einzelnen Mitgliedern steht schon seit einiger Zeit radikal zur Disposition, weil Organisationen heute in einem sehr viel größeren Ausmaß darauf angewiesen sind, ein sehr breites Begabungsrepertoire ihrer Mitglieder zu mobilisieren, damit sie aus sich heraus auf die Prozesse schauen, mitdenken, mitbeobachten, wo Dinge aus dem Ruder laufen. Hier ist die Eigenverantwortung jedes einzelnen heute in einem sehr viel höheren Maße gefragt als dies früher der Fall war. Dieses persönliche Gefragtsein als organisationales Moment trifft zurzeit recht passend auf jene spezifischen Erwartungen, die vor allem die jüngere Generation an die Arbeitswelt mitbringen. 
Hier werden in gewisser Hinsicht Verhältnisse kopiert, wie sie in familiengeführten Unternehmen normal seit jeher sind. Da holt man sich Leute und wenn ihre Persönlichkeitsstruktur eine Passung zeigt zur Kultur des Unternehmens, dann finden sich schon Aufgaben für sie. Und so entwickeln sich dann die Aufgabenverhältnisse in der Organisation um die Personen und ihre Fähigkeiten herum.

Man kann in Einzelfällen natürlich immer begabte Leute hereinholen und dann schauen, wo man sie sinnvoll einsetzen kann. Das macht man ja bei Trainee-Programmen auch so. Aber eine Organisation grundsätzlich um die mitgebrachten Bedürfnisse von Personen herum zu bauen halte ich nicht für erfolgversprechend. Diese Personenorientierung ist ein Organisationsprinzip, das nur bis zu einem gewissen Komplexitätsgrad erfolgreich ist. Außerdem wird damit auf der Personenseite die Illusion genährt, dass die Organisation sich um die eigenen Anliegen und Wünsche herum entwickelt - d.h. die Organisation tritt in den Dienst der Bedürfnisse ihrer Beschäftigten. Diese Illusion hat katastrophale Auswirkungen auf die Organisation. Und Organisationen, die das erfolgreich geschafft haben - im NonProfit-Bereich gibt es viele solcher Organisationen - sind per se veränderungsresistent, weil sie keinen Aufgabenfokus jenseits der persönlichen Bedürfnisse mobilisieren können.

Viele der schnell wachsenden familiengeführten Unternehmen, die eine gewisse Größenordnung erreicht haben, sind heute genau an dieser Stelle gefordert, ihre Organisationsverhältnisse, die vorrangig einer Personenlogik gefolgt sind, in Richtung einer stärkeren Aufgabenlogik umzubauen, die Organisationseinheiten, die dann daraus resultieren, sauber zu konfigurieren und die Führungsverhältnisse dementsprechend anzupassen. Dieser Wechsel von der Personenorientierung in post-patriarchale Führungsverhältnisse ist für viele familiengeführte Unternehmen, gerade für die erfolgreichen, im Moment eine der größten Herausforderungen.

\section{Alle großen Unternehmen versuchen heute agiler zu werden. Ein zentrales Thema ist dabei die Stärkung von Selbstorganisationsmechanismen gegenüber linearen, hierarchischen Steuerungskonzepten. In einem Interview hast du darauf hingewiesen, dass die Umverteilung von Führungsfunktionen an die Basis sinnvoll sein könne, dass man andererseits aber auch die Funktionalität von organisationsspezifischen Strukturen und Einflussunterschieden bedenken müsse. Hast du eine Empfehlung, wie die um eine agilere Arbeitskultur ringenden Organisationen mit diesem Spannungsfeld umgehen können?}

Aus meinen vorherigen Antworten ist, glaube ich, deutlich geworden, dass es für eine Reihe von Herausforderungen, denen sich heutige Unternehmen durch den Zuwachs an Volatilität in ihren relevanten Umwelten ausgesetzt sehen, neue Formen der Arbeitsweltorganisation braucht. Auch jene Unternehmen, die in ihren Geschäftsmodellen nicht so zentral durch die Digitalisierung infrage gestellt werden, benötigen diese neuen Organisationsformen, um das Chancenpotenzial, das mit der Digitalisierung heute verbunden ist, voll auszuschöpfen. Insofern benennt der Kern, der hinter dem Thema Agilität steckt, auch wenn es unter dem Strich ein modischer Hype ist, ein ernstes Entwicklungsproblem unserer Organisationen, das gezielt aufgegriffen und bearbeitet gehört. Eine wesentliche Voraussetzung dafür ist die Anerkenntnis, dass diese neuen Formen der Organisationsgestaltung, in denen die Beteiligten die wesentlichen Führungsaufgaben eigenverantwortlich in Selbstorganisationsprozessen managen lernen sollen, nicht auf die gesamte Organisation ausgedehnt werden können. Das ist ja eine Tendenz, die vor allem in der Beraterliteratur vertreten wird, als ginge es jetzt darum, Organisationen endlich hierarchiefrei zu gestalten und alles, was an Führungserfordernissen da ist, in selbststeuernde, teamförmige Prozesse zu verlagern - Stichwort Laloux, der das ja entsprechend promotet. Und das halte ich für eine absolute Illusion und eine Verkennung dessen, was in Organisationen an Entscheidungserfordernissen da ist, die sich eben nur organisationsförmig bearbeiten lassen und die sich eben nicht in teamförmige Selbststeuerung verlagern lassen. Das heißt, es braucht in manchen Kontexten nach wie vor stabile Über- und Unterordnungsverhältnisse, um überhaupt die heute geforderte Entscheidungsfähigkeit sicherzustellen. Diese Dimension muss man auf der anderen Seite mit agilen, selbststeuernden Formaten zueinander in ein sehr gutes Lebensverhältnis bringen und die Berührungspunkte sauber orchestrieren. 
Die laufende Bewältigung dieser Widerspruchsdynamik ist eine der zentralen Führungsaufgaben, die in der neuen Organisationswelt zu sehen sind.

Wenn man anerkennt, dass es hier auch wie beim Thema Ambidexterity um ein gezieltes Miteinander sehr unterschiedlicher, einander widersprechender Organisationsformen und entsprechender Gestaltungsprinzipien geht, kann man sich überlegen, wie man diese Gleichzeitigkeiten managen kann. Also das, was wir Paradoxiemanagement nennen. Eine der Voraussetzungen dafür ist, dass man ein gutes Gefühl dafür entwickelt, für welche Organisationsthemen, Herausforderungen und Aufgabenfelder solche selbststeuernden, agilen Formate überhaupt sinnvoll sind - und wo sie eben nicht sinnvoll sind.

\section{Ein Großteil der Veränderungsprozesse wird heute von internen Change- Verantwortlichen gesteuert, die aber unternehmensintern oft einen schweren Stand haben. Wie kann aus deiner Sicht das Zusammenspiel von Entscheidungsverantwortung im Management und Prozesskompetenz im internen Change Management im Sinne der Zukunftsfähigkeit von Organisationen sinnvoll gestaltet werden?}

Auch hier muss man sehen, dass das keine gemähte Wiese ist, also kein Selbstläufer. Das war früher schon immer ein sehr schwieriges Spannungsfeld, dem sich interne Change-Experten, Organisationsberaterinnen und -berater ausgesetzt fühlt. Ich erlebe, dass Interne verstärkt unter den Druck geraten, immer wieder zu zeigen, was ihr Mehrwert, ihr Nutzen für die Organisation ist. Und da sind die Entscheidungsträger, aber auch andere in der Organisation, weniger duldsam als früher.

Und durch die aktuellen Entwicklungen sind die Spannungsfelder nicht geringer geworden, im Gegenteil, sie haben noch zugenommen. Ich sehe das Geschäft der internen Berater als eine extrem anspruchsvolle Aufgabe: Sie sind Teil des Organisationsgeschehens und in viele Mechanismen und Muster der Organisation voll mit eingebunden. Nicht nur in kultureller Hinsicht, sondern auch in vielen anderen Aspekten. Und gleichzeitig brauchen sie ausreichend Distanz gegenüber genau jeden problemergänzenden Mustern der Organisation, in die sie eingebunden sind. Nur auf Basis einer solchen Distanzierungsfähigkeit können sie überhaupt wirksam werden und mit ihrer Profession einen Unterschied machen.

Eine zentrale Frage ist die nach der Positionierung gegenüber dem Top-Management. Es ist gut, wenn interne
Berater einen direkten Draht zum Top-Management haben. Gleichzeitig besteht die Gefahr, dass man so zu Erfüllungsgehilfen des Top-Managements wird. In so eine Zuschreibung kommt man relativ rasch und wenn die mal gefestigt ist, kriegt man dieses „Geschmäckle“ als interner Berater fast nicht mehr los. Je intensiver das Vertrauen zum TopManagement ist, umso stärker ist dieses Vertrauen in anderen Ebenen der Organisation gefährdet.

Das sind Paradoxien, die gar nicht leicht zu managen sind und sehr viel emotionale Distanz ermöglichende Selbstreflexion der Akteure brauchen. Aus meiner Sicht haben sich die Widersprüche für das Tätigwerden interner Berater in den letzten Jahren eher verschärft. Ich habe großen Respekt vor all denen, die das als ihre Aufgabe sehen.

Das Interview führte Falko von Ameln im Februar 2019.

Open Access Dieser Artikel wird unter der Creative Commons Namensnennung 4.0 International Lizenz veröffentlicht, welche die Nutzung, Vervielfältigung, Bearbeitung, Verbreitung und Wiedergabe in jeglichem Medium und Format erlaubt, sofern Sie den/die ursprünglichen Autor(en) und die Quelle ordnungsgemäß nennen, einen Link zur Creative Commons Lizenz beifügen und angeben, ob Änderungen vorgenommen wurden.

Die in diesem Artikel enthaltenen Bilder und sonstiges Drittmaterial unterliegen ebenfalls der genannten Creative Commons Lizenz, sofern sich aus der Abbildungslegende nichts anderes ergibt. Sofern das betreffende Material nicht unter der genannten Creative Commons Lizenz steht und die betreffende Handlung nicht nach gesetzlichen Vorschriften erlaubt ist, ist für die oben aufgeführten Weiterverwendungen des Materials die Einwilligung des jeweiligen Rechteinhabers einzuholen.

Weitere Details zur Lizenz entnehmen Sie bitte der Lizenzinformation auf http://creativecommons.org/licenses/by/4.0/deed.de.

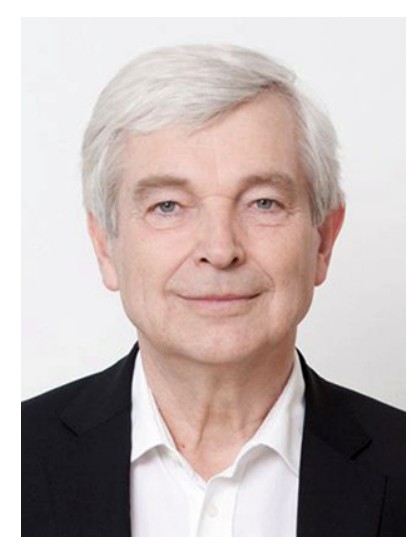

Univ.-Prof. Dr. Rudi Wimmer osb Wien Consulting GmbH Volksgartenstraße 3/1.DG 1010 Wien Austria

PD Dr. Falko von Ameln Leipziger Str. 926506 Norden Germany 